\title{
NARASIMHA: Novel Assay based on Targeted RNA Sequencing to Identify ChiMeric Gene Fusions in Hematological Malignancies
}

\author{
Nikhil Patkar (1) 1,2, Prasanna Bhanshe', Sweta Rajpal', Swapnali Joshi', Shruti Chaudhary', Gaurav Chatterjee ${ }^{1,2}$, \\ Prashant Tembhare 10 1,2, Chetan Dhamne ${ }^{2,3}$, Maya Prasad ${ }^{2,3}$, Nirmalya Roy Moulik²,3, Dhanalaxmi Shetty ${ }^{4}$, \\ Anant Gokarn ${ }^{2,5}$, Avinash Bonda ${ }^{2,5}$, Lingaraj Nayak ${ }^{2,5}$, Sachin Punatkar ${ }^{2,5}$, Bhausaheb Bagal ${ }^{2,5}$, Manju Sengar ${ }^{2,5}$, \\ Gaurav Narula $\mathbb{1}^{2,3}$, Navin Khattry ${ }^{2,5}$, Shripad Banavali, ${ }^{2,3}$, P. G. Subramanian ${ }^{1,2}$ and Sumeet Gujral ${ }^{1,2}$
}

Dear Editor,

Chimeric gene fusions (CGF) are the hallmark of several haematological malignancies. Their exact characterization is critical for accurate diagnosis, administering targeted therapy, as well as effective post therapeutic monitoring. In that context, commonly used molecular techniques such as fluorescent in situ hybridization (FISH) are limited by low sensitivity $(\sim 5-10 \%)$, and an inherent inability to provide sequence level characterization of the chimeric gene. As compared to FISH, real-time PCR (qPCR) is more sensitive but requires a priori nucleotide level knowledge of the CGF. Furthermore, qPCR cannot be multiplexed beyond a few targets and is relatively low throughput in nature. Although transcriptome sequencing has immensely contributed towards the discovery of CGF, its applicability outside of a research setting is limited due to high sequencing costs and an impractical turn-around-time. Researchers have therefore developed focussed target enrichment strategies (or gene-panels) for detection of CGF. Typically, these panels utilize capture probes ${ }^{1,2}$ or multiplexed PCR approaches ${ }^{3,4}$ to enrich targets of interest and detect the CGF, if present. These assays too require prior knowledge of the exact sequence of both partners involved in the formation of CGF thus failing to overcome a principal hurdle of being unable to detect a CGF involving a promiscuous gene where recombination with several partners is known to occur (for e.g., KMT2A-rearranged leukemia) ${ }^{5}$. With recognition of

\footnotetext{
Correspondence: Nikhil Patkar (nvpatkar@gmail.com)

'Haematopathology Laboratory, ACTREC, Tata Memorial Centre, Navi Mumbai, India

${ }^{2}$ Homi Bhabha National Institute (HBNI), Mumbai, India

Full list of author information is available at the end of the article
}

newer entities, such as CGF driven eosinophilia ${ }^{6}$, $B C R-A B L 1$-like $\mathrm{ALL}^{6}$, $\mathrm{B}$-other $\mathrm{ALL}^{7}$ and recent precision medicine initiatives targeting $B C R-A B L 1$-like $\mathrm{ALL}^{8}$, there is an urgent need for developing diagnostic approaches, which are within the reach of most diagnostic laboratories.

We describe NARASIMHA, a targeted RNA-sequencing assay for detection of CGF in blood cancers. NARASIMHA requires knowledge of only one of the partners involved in the formation of a CGF and can detect any potential gene fusion associated with that partner. Sample processing steps include enzymatic fragmentation of second-stranded cDNA followed by end repair, adenylation, and ligation of a novel structure called strand-specific unique molecular motif (spUMM). We designed spUMM to include an eight-base unique molecular identifier (UMI), which results in each strand of a cDNA molecule being tagged with a unique molecular fingerprint. The ligation of a spUMM creates a shotgun assembly where an incomplete semi-Y adapter is attached to each end of cDNA (Fig. 1). A unique aspect of NARASIMHA involves amplification with a primer for target enrichment from one end of cDNA while the other end is amplified with a universal primer when ligated to the spUMM. In subsequent nested PCR steps, a fully functional sequencing ready library is constructed by introduction of sample specific dual indices and instrument-specific adapters. NARASIMHA comprises of independent lymphoid and myeloid modules consisting of different primer sets for target enrichment (Supplementary Tables 1-3). Based on the clinical indication, (acute myeloid leukemia, acute lymphoblastic leukemia, chronic myeloproliferative neoplasm, MDS-MPN, or eosinophilia) we decide upon the NARASIMHA module for library preparation. Details 


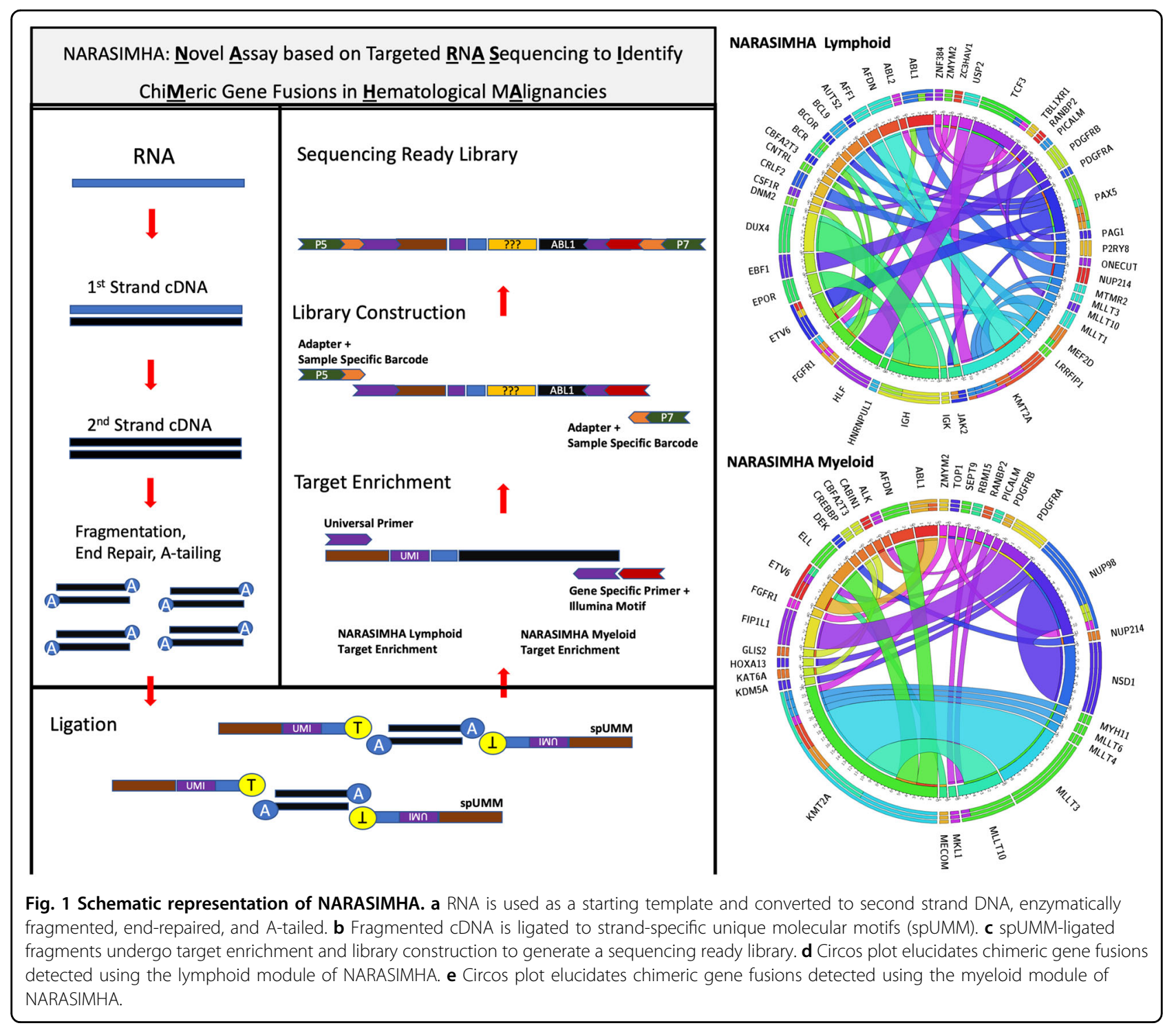

pertaining to assay setup and performance metrics can be seen in supplementary methods accompanying this manuscript. In a linearity experiment, we could demonstrate that both modules detected CGF at a lower limit of $0.5 \%$ (Supplementary Figs. 1 and 2). In initial validation experiments, we demonstrated that NARASIMHA could detect common CGF associated with acute leukemia, such as BCR-ABL1, RUNX1-RUNX1T1, CBFB-MYH11, ETV6RUNX1, and TCF3-PBX1 (Supplemental Methods). Encouraged by this data, we prospectively tested this assay on clinical samples. We describe here a total of 107 CGF detected by NARASIMHA, which would have been difficult (or in some cases impossible) to detect using conventional techniques like FISH and conventional karyotyping. These include five novel CGF as well as other rare CGF. Clinical features, laboratory details including validation of the fusions, flow cytometric MRD, and patient outcome can be seen in Supplementary Tables 4-6 and Supplementary Fig. 3.

The myeloid module of NARASIMHA detected the following CGF (Supplementary Table 5):

(1) AML with KMT2A gene rearrangement $(n=25)$ : These included KMT2A-MLLT3 $(\mathrm{n}=9)$, KMT2AMLLT10 ( $n=6)$, KMT2A-AFDN $(n=3)$, KMT2AELL $(\mathrm{n}=3)$, and one each of KMT2A-MLLT4, KMT2A-MLLT6, KMT2A-MYH11, and KMT2ASEPT9.

(2) AML with NUP98 gene rearrangement $(\mathrm{n}=10)$ : Most commonly this included NUP98-NSD1 $(n=7)$ followed by three others (NUP98-HOXA13, NUP98TOP1, and NUP98-KDM5A).

(3) Other rare CGF seen in AML $(n=5)$ : These included entities recognized recently by the WHO classification, such as AML with ETV6-MECOM $(n=1)$, AML 
with RBM15-MKL1 $(\mathrm{n}=1)$, and AML with DEKNUP214 $(n=1)$. Other rare fusions, such as KAT6A$\operatorname{CREBBP}(\mathrm{n}=1)$ and CBFAT-GLIS2 $(\mathrm{n}=1)$, were also observed.

(4) Myeloproliferative neoplasms including molecular workup of unexplained eosinophilia $(n=9)$ : CGF in patients with myeloproliferative neoplasms included CABIN1-ABL1 $(n=1)$, ETV6-ABL1 $(n=2)$, ETV6PDGFRB $(\mathrm{n}=1), \quad$ ZMYM2-FGFR1 $(\mathrm{n}=1)$, and FIP1L1-PDGFRA $(\mathrm{n}=4)$.

(5) Miscellaneous: One patient with mixed lineage acute leukemia demonstrated NUP98-NSD1 fusion, whereas we also observed a rare RANBP2-ALK CGF in a case of chronic myelomonocytic leukemia with eosinophilia (which did not have any other somatic mutation). A patient of acute leukemia of ambiguous lineage harboured PICALM-MLLT10.

The lymphoid module of NARASIMHA detected 51 CGF (excluding novel rearrangements described in Supplementary Table 4) as a result of which we could classify patients under these broad categories (Supplementary Table 6):

(1) BCR-ABL1-like BCP-ALL: We detected 18 patients with BCR-ABL1-like BCP-ALL. These included cases with rearrangements of ABL1 ( $\mathrm{n}=$ 3; NUP214, ETV6, RANBP2), ABL2 $(\mathrm{n}=1$; ZC3HAV1), CRLF2 ( $\mathrm{n}=2$; P2RY8-CRLF2), EPOR $(\mathrm{n}=3$; EPOR-IGH), FGFR1 $(\mathrm{n}=3$, BCR, CNTRL, LRRFIP1), JAK2 ( $\mathrm{n}=2$; BCR, PAX5), CSF1R $(\mathrm{n}=$ 1; TBL1XR1), and PDGFRB ( $\mathrm{n}=3$; EBF1).

(2) B-other BCP-ALL: A total of 21 patients had CGF that could be classified as B-other BCP-ALL. Most commonly these harboured PAX5 rearrangement $[\mathrm{n}=6$; ETV6 $(\mathrm{n}=3)$, AUTS2, BCOR, CBFA2T3(1 each)], TCF3 [(TCF3-HLF $(\mathrm{n}=5)$; TCF3-ONECUT3 $(\mathrm{n}=1)$ ], DUX4 $(\mathrm{n}=5$; DUX4-IGH), MEF2D [BCL9 $(\mathrm{n}=2)$, HNRNPUL1 $(\mathrm{n}=1)]$, and ZNF384 $(\mathrm{n}=1$; TCF3-ZNF384).

(3) KMT2A-rearranged BCP-ALL: These included KMT2A-AFF1 $(\mathrm{n}=2), \quad$ KMT2A-USP2 $\quad(\mathrm{n}=2)$, KMT2A-MLLT1 $(\mathrm{n}=1)$, and KMT2A-MLLT3 $(\mathrm{n}=1)$.

(4) Precursor T Lineage ALL (T-ALL): We observed four patients with KMT2A rearrangements [KMT2AAFDN, $(n=2)$; KMT2A-MTMR2 $(n=1)$, KMT2A$\operatorname{MLLT1}(\mathrm{n}=1)$ ] of which three harboured an ETPALL immunophenotype. We also observed ZMYMFGFR1, NUP214-ABL1, IGK-DUX4, ETV6-DNM2, and PAG1-PDGFRA in five patients of T-ALL (Supplementary Tables 4 and 6).

(5) Miscellaneous: One cases of acute leukemia of ambiguous lineage harboured PICALM-MLLT10 and another harboured KMT2A-AFDN.

Novel CGF were detected in five patients (Supplementary Table 4).
(1) MTMR2-KMT2A: In-frame fusion was observed between exon 8 of KMT2A and exon 3 of the MTMR2 gene in a 31-year-old male with ETP-ALL. This is a novel partner of the KMT2A recombinome.

(2) ETV6-DNM2: Out-of-frame fusion seen between exon 2 of ETV6 and exon 13 of DNM2. Novel partner of ETV6 in a 15-year-old male with T-ALL.

(3) PAG1-PDGFRA: Novel partner of PDGFRA where the 5' UTR of PAG1 is involved in fusion with exon 10 of PDGFRA in a 30-year-old male with ETP-ALL.

(4) TCF3-ONECUT3: In-frame fusion in a 10-year-old child with BCP-ALL between exon 16 of TCF3 and exon 2 of ONECUT3.

(5) IGK-DUX4: DUX4 associated translocation was seen with a novel partner (IGK@) in a 23-year-old male with T-ALL.

We could validate each of the above described CGF using orthogonal techniques, such as RT-PCR and/or FISH (Supplementary Tables 4-6 and Supplementary Fig. 4). A total of $35 K M T 2 A$ rearrangements were detected by NARASIMHA. Of these, 28 were detected as $K M T 2 A$ rearranged by FISH (partner could not be identified in 18; partner characterized in 10). In six cases FISH missed the KMT2A rearrangement. In one case FISH was not performed.

Previously, Zheng et al. have previously described an anchored multiplex PCR assay that enable a user to detect CGF without prior knowledge of fusion partners ${ }^{9}$. As compared to Zheng's method NARASIMHA represents a technical advance by the inclusion of an UMI. This enables us to perform absolute cDNA molecule counting and reduction of PCR bias as every molecule of cDNA is marked uniquely by a random oligonucleotide. Recently, Dillon et al. described a method for ultrasensitive MRD monitoring of $\mathrm{CGF}^{10}$. They performed multiplexed cDNA synthesis of a limited number of targets and incorporated a UMI using PCR after the cDNA synthesis stage. As compared to NARASIMHA, Dillon's assay is more sensitive but is unable to detect unknown partners of CGF.

The power of NARASIMHA lies in the fact that we can detect CGF that are challenging for conventional techniques, such as BCR-ABL1-like ALL, B-other BCP-ALL, KMT2Arearranged malignancies as well as cryptic lesions that will be missed by FISH (for e.g., NUP98-NSD1, DUX4 rearrangements, KMT2A-USP2 fusions). The clinical potential of this assay is evident from our data on prospective testing. This assay could contribute to the diagnosis of difficult cases (such as $B C R-A B L 1$-negative myeloproliferative neoplasms as well as cases with unexplained eosinophilia) and enable appropriate prognostication by delineating extremely high risk acute leukemia (for e.g., TCF3-HLF-rearranged BCP-ALL or NUP98-NSD1-rearranged AML). The clinical utility of this assay in detection of $B C R-A B L 1$-like ALL cannot be overstated. Nearly $93 \%$ of cases in which FCM-MRD testing was performed (13 out 14 cases; Supplementary Table 6) were 
high MRD positive (median 7.6\%) at end of induction indicating a generally poor outcome for this disease. The singular case that was MRD-negative was treated with dasatinib in addition to conventional ALL chemotherapy. Although this is not explicitly demonstrated here, this assay has the potential to track MRD by monitoring CGF. This can be made possible by using high-throughput sequencers, such as the Illumina NextSeq 550 or beyond.

The cost of library preparation is approximately (USD) $\$ 56$. The cost of sequencing is $\$ 70$ for 2 million reads on a MiSeq v2- 500 cycle chemistry. The turn-around time for our test is 2 weeks. Unlike commercial assays (or for that matter even some of the previously published papers, which do not reveal their methodology $)^{9}$, we offer an open source solution that will reduce the cost of testing and enable laboratories to develop customized solutions. Importantly, we provide an assay that could enable the implementation of precision medicine in diseases like $B C R-A B L 1$-like ALL and enable laboratories to meaningfully classify diseases beyond the WHO 2017 classification. We estimate that incorporation of NARASIMHA into routine diagnostic workflows will make conventional techniques such as FISH redundant.

\section{Acknowledgements}

We thank Dr David Wu, Department of Laboratory Medicine, University of Washington for his helpful discussions and critical inputs. This work was supported by the Wellcome Trust/DBT India Alliance Fellowship [grant number IA/CPHI/14/1/501485] awarded to Dr Nikhil Patkar.

\section{Author details}

${ }^{1}$ Haematopathology Laboratory, ACTREC, Tata Memorial Centre, Navi Mumbai, India. ${ }^{2}$ Homi Bhabha National Institute (HBNI), Mumbai, India. ${ }^{3}$ Pediatric Haematolymphoid Disease Management Group, Tata Memorial Centre, Mumbai, India. ${ }^{4}$ Department of Cytogenetics, ACTREC, Tata Memorial Centre, Navi Mumbai, India. ${ }^{5}$ Adult Haematolymphoid Disease Management Group, Tata Memorial Centre, Mumbai, India
Conflict of interest

The authors declare that they have no conflict of interest.

\section{Publisher's note}

Springer Nature remains neutral with regard to jurisdictional claims in published maps and institutional affiliations.

Supplementary Information accompanies this paper at (https://doi.org/ 10.1038/s41408-020-0313-6).

Received: 31 January 2020 Revised: 9 April 2020 Accepted: 17 April 2020 Published online: 05 May 2020

\section{References}

1. Heyer, E. E. et al. Diagnosis of fusion genes using targeted RNA sequencing. Nat. Commun. 10, 1388 (2019).

2. Reeser, J. W. et al. Validation of a targeted RNA sequencing assay for kinase fusion detection in solid tumors. J. Mol. Diagn. 19, 682-696 (2017).

3. Blidner, R. A. et al. Design, optimization, and multisite evaluation of a targeted next-generation sequencing assay system for chimeric RNAs from gene fusions and exon-skipping events in non-small cell lung cancer. J. Mol. Diagn. 21, 352-365 (2019).

4. Ruminy, P. et al. Multiplexed targeted sequencing of recurrent fusion genes in acute leukaemia. Leukemia 30, 757-760 (2016).

5. Mertens, F., Johansson, B., Fioretos, T. \& Mitelman, F. The emerging complexity of gene fusions in cancer. Nat. Rev. Cancer 15, 371-381 (2015).

6. Arber, D. A. et al. The 2016 revision to the World Health Organization classification of myeloid neoplasms and acute leukemia. Blood 127, 2391-2405 (2016).

7. Schwab, C. \& Harrison, C. J. Advances in B-cell precursor acute lymphoblastic leukemia genomics. Hemasphere 2, e53 (2018).

8. Tanasi, I. et al. Efficacy of tyrosine kinase inhibitors in Ph-like acute lymphoblastic leukemia harboring ABL-class rearrangements. Blood 134, 1351-1355 (2019).

9. Zheng, Z. et al. Anchored multiplex PCR for targeted next-generation sequencing. Nat. Med. 20, 1479-1484 (2014).

10. Dillon, L. W. et al. Targeted RNA-sequencing for the quantification of measurable residual disease in acute myeloid leukemia. Haematologica 104, 297-304 (2019). 